\title{
Fluctuations of monatomic steps on $\mathrm{Si}(001)$
}

\author{
H. J. W. Zandvliet and B. Poelsema \\ Faculty of Applied Physics, University of Twente, P.O. Box 217, 7500 AE Enschede, The Netherlands \\ H. B. Elswijk \\ Philips Research Laboratories, P.O. Box 80000 , 5600 JA Eindhoven, The Netherlands
}

(Received 5 July 1994)

\begin{abstract}
The motion of monatomic steps on $\mathrm{Si}(001)$ is studied on an atomic scale at elevated temperatures with scanning tunneling microscopy. The kinks in the step edges move in units of two dimers along the monatomic $A$-type step edge and perpendicular to the monatomic $B$-type step edge. The overall time dependencies of the equilibrium step fluctuations of $A$ - and $B$-type step edges were found to be both proportional to $t^{0.6 \pm 0.1}$. The fluctuations of long kinks in the $B$-type step edge are, however, much larger and exhibit initially a linear $t$ dependence, i.e., one-dimensional random-walk behavior. Both time dependencies can be understood in terms of the Langevin equation.
\end{abstract}

Step edges on surfaces play an important role in epitaxial growth and adsorption processes. From a more fundamental point of view, step edges are also challenging because they play a key role in many surface thermodynamic problems like roughening and faceting. ${ }^{1,2}$ In most cases, step edges are unavoidable because it is virtually impossible to cut a crystal exactly along one of its low Miller indices planes. The slight misorientation that usually results must be accommodated with surface steps. The exact time dependence of step fluctuation has been a topic of interest in several theoretical ${ }^{3,4}$ as well as experimental papers. ${ }^{5-12}$ Bartelt et al. $^{3}$ and Pimpinelli et al. ${ }^{4}$ have shown that depending on the exact mechanism of mass transfer, different time dependencies can be expected. In the case when step motion occurs through the exchange of atoms with a reservoir of adatoms on the terraces the equilibrium step fluctuation is proportional to the square root of time, whereas it goes as the one fourth power of the time in the case when the motion of the steps occurs only through diffusion along the edges. If the adatom diffusion rate on the terraces is, however, slow compared to the adatom emission rate from the steps the step fluctuations become proportional to the one third power of time. In this specific case, a crossover to a $\sqrt{t}$ dependence is expected when the step separation is sufficiently small.

Recent experimental work has revealed that in the case of $\mathrm{Si}(111)$ (Ref. 5) and $\mathrm{Au}(110)$ (Ref. 6) the step fluctuations are proportional to the square root of time, whereas they are proportional to the one fourth power of time for vicinal $\mathrm{Cu}(001)$ surfaces. ${ }^{7}$ Another very interesting paper, in this context, which should certainly be mentioned is that by Kitamura et al. ${ }^{9}$ Kitamura et al. ${ }^{9}$ analyzed the step fluctuations of a vicinal $\mathrm{Si}(001)$ on a time scale which was significantly smaller than the typical time lapse between successive detachment/attachment events $(\tau)$. The measured probability distribution of the positional change of the step edge after a time lapse of $13 \mathrm{~s}[\tau \approx 40 \mathrm{~s}$ (Ref. 9)] indicates that the step segments perform a onedimensional random walk. This random-walk behavior is in agreement with predictions of Abraham and Upton, ${ }^{13}$ who have shown that there is a crossover from a linear $t$ dependence for times smaller than $\tau$ (the exact position of the crossover depends on the strength of the interaction between the neighboring steps edge atoms ${ }^{13}$ ) to $\sqrt{t}$ for times larger than $\tau$.

It is the aim of this paper to study the time dependence of the step fluctuations on short-time scales, i.e., a time scale ranging typically from a few times $\tau$ up to about $30 \tau$. Besides the already mentioned possible time dependencies of the equilibrium step fluctuations, vicinal pinned parts of the step edge (which contain often several long kinks) exhibit initially a random-walk-like behavior on a time scale, which goes significantly beyond the typical crossover time, i.e., $\approx \tau$. It will be shown that this time dependence can easily be understood by making contact with the Langevin equation. In our study, we have focused our attention mainly on protruding parts of the step edge. This was for two reasons: first, protruding segments are easy to follow in subsequent images even if the STM suffers from a significant thermal drift and relatively long scanning times and second especially these parts of the step edge fluctuate most rapidly.

The $\mathrm{Si}(001)$ samples were cut from commercially available wafers (Wacker, floating zone B-doped $8-12 \Omega \mathrm{cm}$ ) and ultrasonically rinsed in ethanol before loading them into the vacuum system. The sample and holder were subsequently outgassed inside of the vacuum system with a base pressure of $1 \times 10^{-10}$ Torr at a temperature of about $700 \mathrm{~K}$ for several hours. In order to obtain an atomically clean surface, the samples were heated resistively up to a temperature of about $1500 \mathrm{~K}$ for several seconds. During cleaning, the pressure was maintained below $1 \times 10^{-9}$ Torr in order to avoid contamination of the sample surface. This procedure results in a twodomain $(2 \times 1)$ reconstructed atomically clean $\mathrm{Si}(001)$ surface with a few percent of a monolayer of missing dimers as the most prominent defects (see Fig. 1). A hightemperature scanning tunneling microscope (STM), described in Ref. 8, is used to image the $\mathrm{Si}(001)$ surface at elevated temperatures up to about $800 \mathrm{~K}$. The modifications of the high temperature STM as compared to a normal STM are a Ta heat shield mounted between the sample and the $x y z$-piezo scanner, with the tip wire protruding through a small hole in the shield and an additional electrical lead towards the sample in order to 


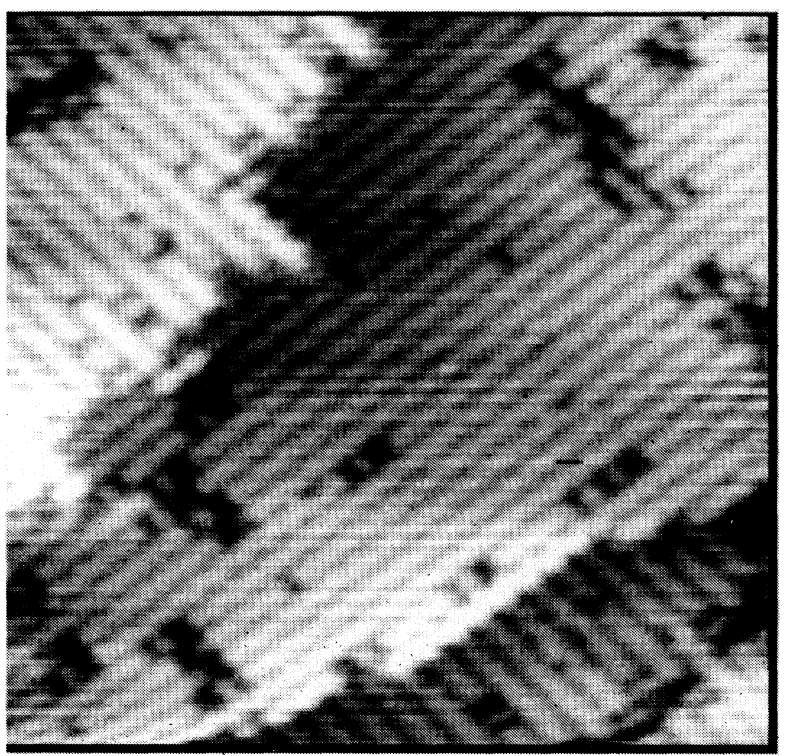

FIG. 1. A typical room-temperature STM image of a vicinal $\mathrm{Si}(001)$ surface taken at $-2-\mathrm{V}$ sample bias and $0.5-\mathrm{nA}$ tunneling current. Scan size $40 \times 40 \mathrm{~nm} .^{2}$

heat it resistively. A resistive divider in parallel with the sample is used to provide a tip-sample bias voltage in an approximately symmetric way. Most of the STM images are taken with a sample bias of -1.5 or $-2 \mathrm{~V}$ and a tunneling current of $0.5 \mathrm{nA}$.

The $\mathrm{Si}(001)$ surface reconstructs by forming surface dimers that are arranged in parallel rows. The dimers can be oriented along two possible directions, depending on the plane where the crystal is cut. Hence the surface has two degenerate phases, which are related by a $90^{\circ}$ rotation; their surface periodicity is either $2 \times 1$ or $1 \times 2$. Two distinct monatomic types of step edges, denoted $S_{A}$ and $S_{B}$, can be found on a vicinal $\mathrm{Si}(001)$ surface. The difference in the two is that the direction of the dimer rows of the upper terrace is parallel $\left(S_{A}\right)$ or perpendicular $\left(S_{B}\right)$ to the direction of the step edge. Due to the symmetry of the silicon lattice a monoatmic step edge is always a phase boundary between $2 \times 1$ and $1 \times 2$ reconstructed domains. Kinks in monatomic step edges of $\mathrm{Si}(001)$ tend to offset the step edge in a direction perpendicular to the edge by $2 a(=7.7 \AA$, the width of a dimer row) or multiples of $2 a$. In principle there are two different $S_{B}$ steps edges, which can occur depending on whether the dimer row of the upper terrace ends just on a dimer row of the lower terrace or just in between two dimer rows of the upper terrace. The latter configuration gives rise to rebonding, i.e., a reduction of dangling bonds, whereas the former configuration is characterized by having a dangling bond on each second-layer edge atom. Energy calculations of $\mathrm{Chadi}^{14}$ have shown that the nonbonded is not as energetically favorable as the rebonded configuration. Scanning-tunneling-microscopy studies of vicinal $\mathrm{Si}(001)$ surfaces reveal that indeed the rebonded configuration is the dominant one, although occasionally, also, the nonbonded configuration has been observed. ${ }^{15}$ The preference for just one of the two possi- ble step structures make it possible for almost all kink lengths to have a length which is $2 a$ or a multiple of $2 a$. A monoatomic $S_{B}$ step edge can be represented by an array of integers specifying the number of dimer pairs in each column (dimer row) perpendicular to the step edge, or equivalently by the length of the column $\left(h_{i}\right)$ relative to the flat $T=0$ reference step edge. The blocks of two dimers in the columns interact with their neighboring blocks in adjacent rows or within the same row with an interaction energy $\varepsilon$ of about 0.24 and $0.38 \mathrm{eV}$, respectively. ${ }^{16,17}$

Figure 2 shows a sequence of STM images of a vicinal $\mathrm{Si}(001)$ surface taken at a temperature of about $725 \mathrm{~K}$. The time lapse between the images varies a little but is typically of the order of $1 \mathrm{~min}$. After comparing the subsequent images with each other, several remarkable features, some of which have already been discussed in Refs. 8 and 9, emerge immediately.

(i) The position of the step edge often changes from one image to the next image. The modifications occur most frequently at kink sites which are not attached to missing dimer defects on the lower terrace. The observed events involve at least a unit of two dimers or multiples of this unit. $^{8,9}$

(ii) The rapid kink movement is in a direction perpendicular to the $S_{B}$ step edge and parallel to the $S_{A}$ step edge.

(iii) Kinks attached to missing dimer defects on the lower terrace do not change their position at this temperature on a time scale of at least 15 min. ${ }^{8}$

(iv) The averaged step fluctuations of long kink sites (somewhat arbitrary we call a kink a long kink when the distance to its neighbor kinks in both adjacent dimer rows is longer than or equal to four dimer row spacings, i.e., about $30 \AA$ ) appear to be larger as compared to short kink sites.

(v) Occasionally dimer rows break up spontaneously in segments giving rise to additional kink sites.

We now consider, in somewhat more detail, the exact time dependence of the step fluctuations. Since we want to understand how a configuration $\{h\}$ relaxes to equilibrium, we use the Langevin equation of motion,

$$
\begin{aligned}
& \frac{\partial h_{j}}{\partial t}=-\left(\tau k_{b} T\right)^{-1} \frac{\partial H\{h\}}{\partial h_{j}}+\eta_{j}, \\
& \left\langle\eta_{j}(t)\right\rangle=0, \\
& \left\langle\eta_{j}(t) \eta_{i}\left(t^{\prime}\right)\right\rangle=2 \tau^{-1} \delta_{i j} \delta\left(t-t^{\prime}\right),
\end{aligned}
$$

where $H\{h\}$ refers to the Hamiltonian (actually a free energy since the internal degrees of freedom have been summed out) and $\eta_{j}(t)$ to a Gaussian white noise at site $j$. The first term in the right-hand side of Eq. (1) mimics the desire of the step edge to relax to a situation where the free energy is minimal and $\eta$ is a noise term which gives rise to thermal fluctuations. The presence of the "friction coefficient" $\tau^{-1}$ ensures that in the static limit, the correct equilibrium distribution will be obtained.

The relaxation dynamics of an initially flat line in a two-dimensional system, modeled by an unweighted Gaussian Hamiltonian and obeying Langevin dynamics, 
was studied by Abraham and Upton. ${ }^{13}$ In their study, they found that the averaged fluctuations in the position of the line were proportional to $t$ for short times $(t<\tau)$ and proportional to the square root of the time for time lapses, which go beyond a few times $\tau$.

In order to interpret our experimental results, we consider here only the very simple case of a small piece of a step edge $\left(S_{B}\right)$ trapped between two pinning centers. Furthermore, all interactions which go beyond the nearest neighbors are omitted. The Hamiltonian can be written as

$$
H=\frac{1}{2} \varepsilon \sum_{j}\left|h_{j+1}-h_{j}\right|,
$$

where $\varepsilon$ is the nearest-neighbor interaction energy $(\approx 0.24 \mathrm{eV}$, see Refs. 16 and 17 ). Between the pinning
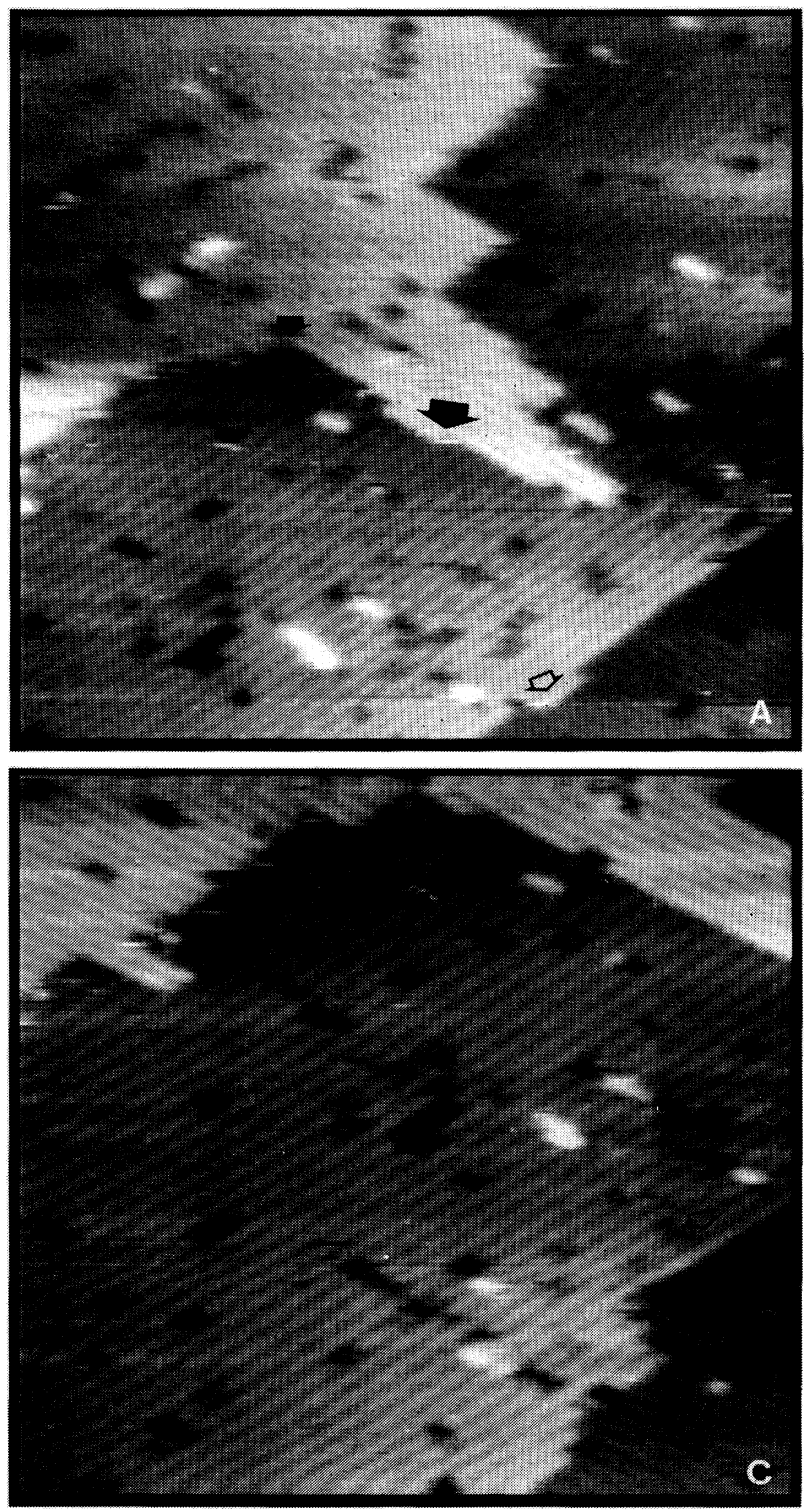

centers, which are assumed to be at different positions ( $h_{-1}=0$ and $h_{+1}=M$ ), there is only one column, being $h_{0}$, which may change its position. By requiring that $h_{0}$ is initially positioned at $L(0<<<M / 2)$, it is easy to verify that $\left\langle h_{0}^{2}\right\rangle$ equals $2 \tau^{-1} t$ [the Langevin equation reduces in this special case, where $\partial H / \partial h=0$, simply to $\partial h / \partial t=\eta(t)]$. This one-dimensional (1D) random-walk behavior breaks down as soon as the fluctuations start to reach the closest pinning centers, i.e., $2 \tau^{-1} t=L^{2} \cdot{ }^{18,19}$ In Fig. 3, the step fluctuations of $S_{A}$ and $S_{B}$ step edges and long kinks are shown separately in a log-log plot. The two solid lines in Fig. 3 serve as a reference and have slopes of 1 or $\frac{1}{2}$ referring to the random walk (i.e., $t$ dependence) and $\sqrt{t}$ dependence of the step fluctuations, respectively. Although the statistics (each time data point is averaged over about $100-300$ points) and the
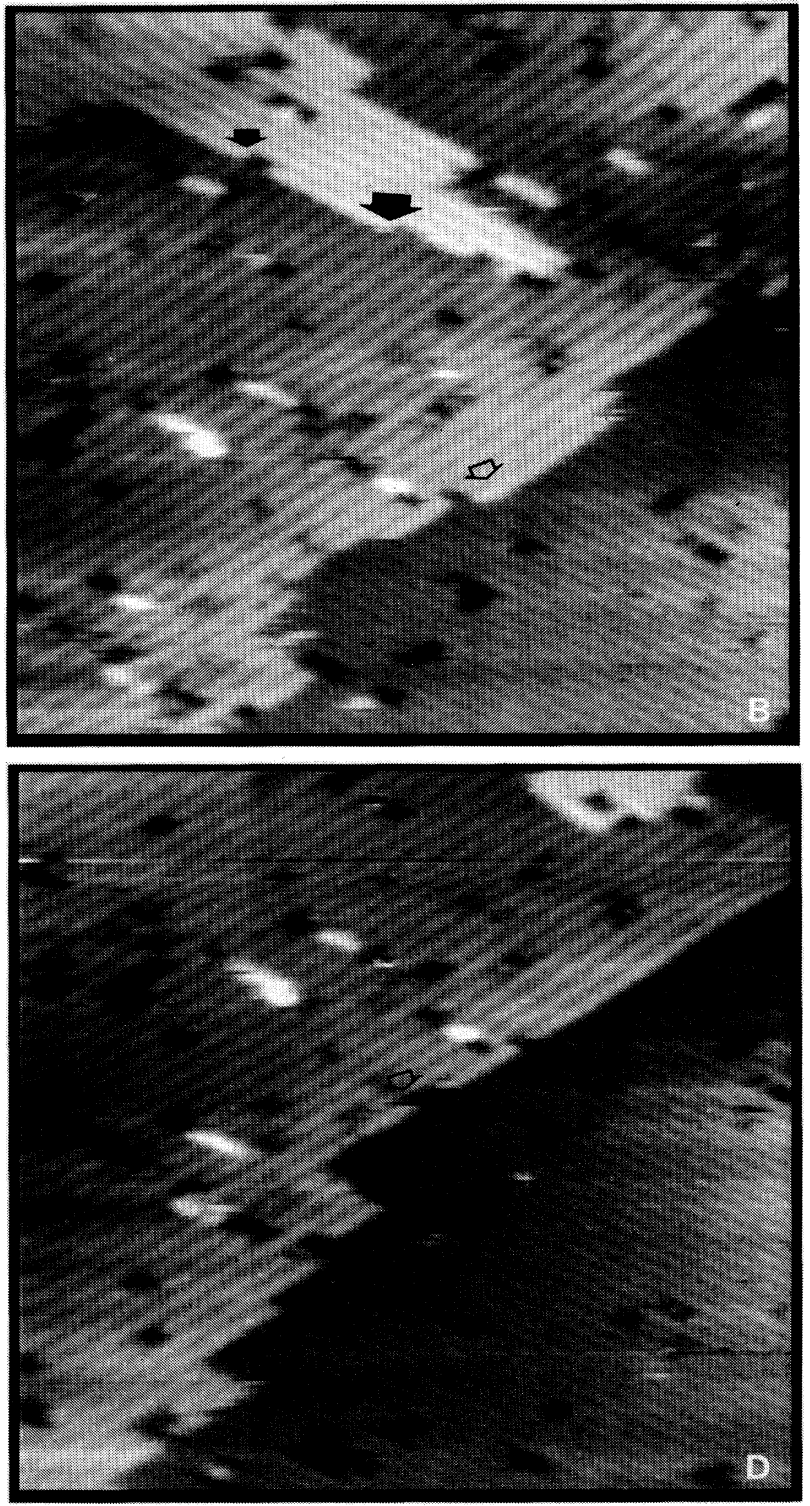

FIG. 2. (a)-(d) Several subsequent STM images of a vicinal $\mathrm{Si}(001)$ surface, at a temperature of about $725 \mathrm{~K}$. The sample bias was $-1.5 \mathrm{~V}$ and the tunneling current was $0.5 \mathrm{nA}$. The typical time lapse between successive images is about 1 min. Scan size $30 \times 30$ $\mathrm{nm} .^{2}$ 


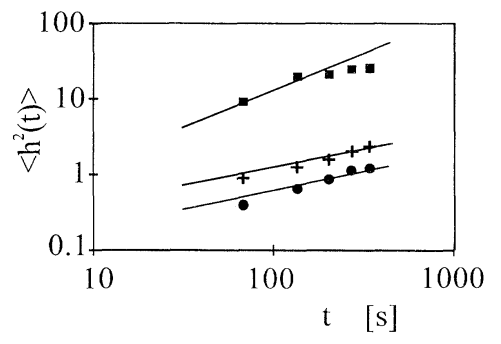

FIG. 3. Log-log plot of the equilibrium step fluctuations, $\left\langle h^{2}(t)\right\rangle$, versus time at about $725 \mathrm{~K}$. (D) Behavior of extremely long kinks $(L \geq 4)$. ( +$) S_{B}$ step edge ( with $\left\langle k^{2}\right\rangle \approx 1.5$ ). (O) $S_{A}$ step edge (with $\left\langle k^{2}\right\rangle \approx 0.2$ ). The solid lines serve to guide the eye. The lines have slopes 1 or $\frac{1}{2}$ and refer to $1 \mathrm{D}$ random behavior (i.e., linear $t$ dependence) and $\sqrt{t}$ dependence of the step fluctuations, respectively.

number of time data points are insufficient to determine the exact time dependence, the difference between normal kinks and long kinks is immediately clear. From a straightforward analysis Bartelt et al., ${ }^{3}$ Abraham and Upton $^{13}$ and Poensgen et al. ${ }^{10}$ have shown independently that for the case of step motion limited by the mass transfer between the terraces and the step edges, the step fluctuations should be proportional to $\sqrt{t}$,

$$
\left\langle h^{2}(t)\right\rangle=\left(2\left\langle k^{2}\right\rangle \tau^{-1} t / \pi\right)^{1 / 2} .
$$

In principle, Eq. (3) should be valid on time scales larger than several attachment/detachment events, but short compared to the typical time period between step edge collisions. The typical time period between successive attachment/detachment events, $\tau$, as extracted from the random-walk behavior of the long kinks of both monatomic step edges is about $16 \mathrm{~s}$. The prefactors of the curves with a slope of $\frac{1}{2}$ results in $\left\langle k^{2}\right\rangle$ values of about 0.1 for the $S_{A}$ step and about 0.5 for the $S_{B}$ step. For the straight $S_{A}$ and rough $S_{B}$ step edges, we have analyzed in this specific study, however, $\left\langle k^{2}\right\rangle$ is determined to be about 0.2 and 1.5, respectively (these values are in good accordance with Refs. 16 and 17). The discrepancy of a factor 2-3 between calculated values of $\left\langle k^{2}\right\rangle$ using Eq. (3) and the experimentally determined values as extracted from the step edge roughness is, however, still unclear for us.

For the long kinks there is, as expected, a crossover from the one-dimensional random walk to $\sqrt{t}$ dependence around $t \approx 5-10 \tau\left(L \geq 4\right.$ and $\left.\left.\angle k^{2}\right\rangle \approx 40\right)$. It is important to note that our $\mathrm{Si}(001)$ surface contains a relatively high density of missing dimer defects which give rise to step pinning and, consequently, to a relatively high density of such long kinks because the pinned segments make often a large azimuthal angle with respect to the mean direction of the edge itself. For steps completely free of pinning centers the density of long kinks, $n_{l k}$, is usually very small [for a relatively defect free $S_{B}$ monatomic step edge $n_{l k}$ is typically about 0.04 (Ref. 17)]. In order to improve the statistics of the long kinks, we have also analyzed the fluctuations of kinks in $S_{A}$ step edges measured along the step edge instead of perpendicular to the step edge as in the case of the monatomic $S_{B}$ step edges. As has been pointed out by Poensgen et al. ${ }^{10}$ and Kuipers, Hoogeman, and Frenken ${ }^{6}$ the $1 \mathrm{D}$ random walk of kinks along the step edge gives rise to a $\sqrt{t}$ dependence for the step fluctuation measured perpendicular to the step edge in agreement with our experimental observations. The $\sqrt{t}$ dependence for fluctuating monatomic step edges on $\mathrm{Si}(001)$ has also been observed by Bartelt ${ }^{20,21}$ using the low-energy-electron-microscopy data of Tromp. Bartelt et al. ${ }^{3}$ have also clearly shown, in a separate theoretical paper that, in this particular case, step motion must occur through an exchange of atoms with the terraces.

In summary, the averaged equilibrium step fluctuations of monatomic step edges on vicinal $\mathrm{Si}(001)$ are proportional to $t^{0.6 \pm 0.1}$. Strongly misoriented parts of the step edge exhibit initially, however, a 1D random-walk-like behavior (i.e., a linear $t$ dependence). Both time dependencies can be understood in terms of the Langevin equation. In the former case this is an indication that the step motion is due to an exchange mechanism with adatoms on the terraces, whereas in the latter case only the noise term in the Langevin equation is actually probed.
${ }^{1}$ E. D. Williams and N. C. Bartelt, Science 251, 393 (1991).

${ }^{2}$ E. D. Williams, Surf. Sci. 299/300, 502 (1994).

${ }^{3}$ N. C. Bartelt et al., Surf. Sci. 273, 252 (1992).

${ }^{4}$ A. Pimpinelli et al., Surf. Sci. 295, 143 (1993).

${ }^{5}$ N. C. Bartelt et al., Phys. Rev. B 48, 15453 (1993).

${ }^{6}$ L. Kuipers et al., Phys. Rev. Lett. 71, 3517 (1993).

${ }^{7}$ M. Giesen-Seibert et al., Phys. Rev. Lett. 71, 3521 (1993).

${ }^{8}$ H. J. W. Zandvliet et al., Surf. Sci. 272, 264 (1992).

${ }^{9}$ N. Kitamura et al., Phys. Rev. B 48, 5704 (1993).

${ }^{10}$ M. Poengsen et al., Surf. Sci. 274, 430 (1992).

${ }^{11}$ M. Giesen et al., J. Vac. Sci. Technol. A 10, 2597 (1992).

12J. C. Girard et al., Surf. Sci. 301, 245 (1994).

${ }^{13}$ D. B. Abraham and P. J. Upton, Phys. Rev. B 39, 736 (1989).

${ }^{14}$ D. J. Chadi, Phys. Rev. Lett. 59, 1691 (1987).

${ }^{15}$ R. J. Hamers et al., Phys. Rev. B 34, 5343 (1986).

${ }^{16}$ B. S. Swartzentruber et al., Phys. Rev. Lett. 65, 1913 (1990).

${ }^{17}$ H. J. W. Zandvliet et al., Phys. Rev. B 45, 5965 (1992).
${ }^{18}$ One expects that the random-walk behavior breaks down as soon as the fluctuations become of the order of the meansquare displacement of the kinks $\left\langle k^{2}\right\rangle$, i.e., $2 \tau^{-1} t \approx\left\langle k^{2}\right\rangle$, where $\quad\left\langle k^{2}\right\rangle=\sum k^{2} \exp \left(-\varepsilon_{k} / k_{b} T\right) / \sum \exp \left(-\varepsilon_{k} / k_{b} T\right)$ $=2 \exp \left(-\varepsilon / 2 k_{b} T\right) /\left(1-\exp \left(-\varepsilon / 2 k_{b} T\right)^{2}, \varepsilon_{k}=k \varepsilon / 2\right.$ the kink formation energy of a kink with length $k$, and $\varepsilon$ the nearestneighbor interaction energy.

${ }^{19}$ In the case that the kink position is allowed to pass its neighboring pinning centers, a crossover to a $\sqrt{t}$ dependence is expected; see Ref. 13 for the details. If, however, the kink site cannot cross the neighbor pinning sites, the step fluctuation, $\left\langle h^{2}(t)\right\rangle$, will finally saturate at $\left(1 / 6-1 / \pi^{2}\right) M^{2}$ for large $t$ (we assume for simplicity that the only interaction between the kink site and pinning center is the noncrossing restriction).

${ }^{20}$ N. C. Bartelt, R. M. Tromp, and E. D. Williams, Phys. Rev. Lett. 73, 1656 (1994).

${ }^{21}$ N. C. Bartelt (private communication). 


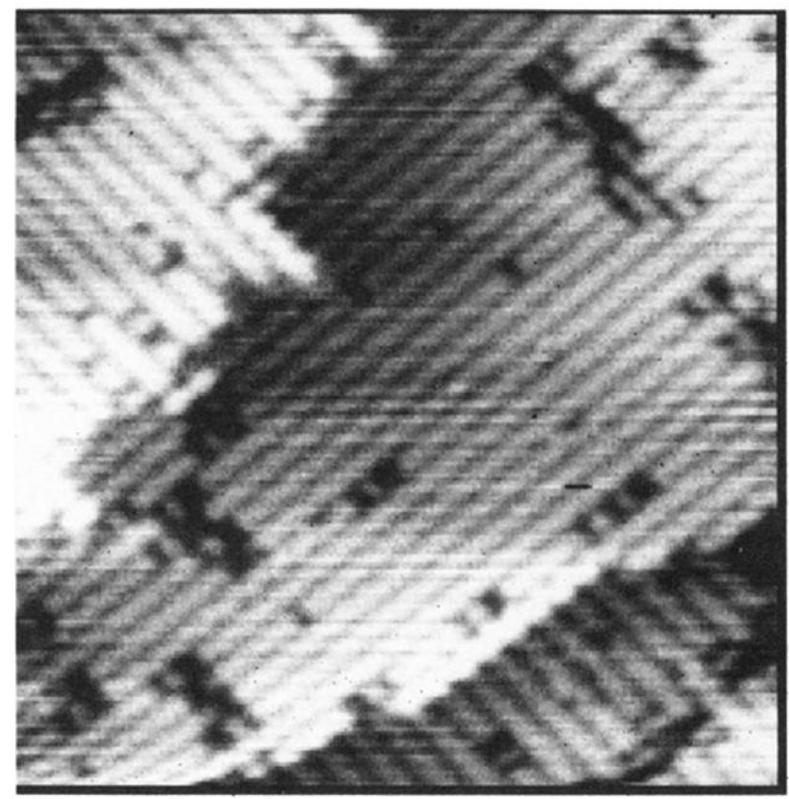

FIG. 1. A typical room-temperature STM image of a vicinal $\mathrm{Si}(001)$ surface taken at $-2-\mathrm{V}$ sample bias and $0.5-\mathrm{nA}$ tunneling current. Scan size $40 \times 40 \mathrm{~nm}^{2}$ 

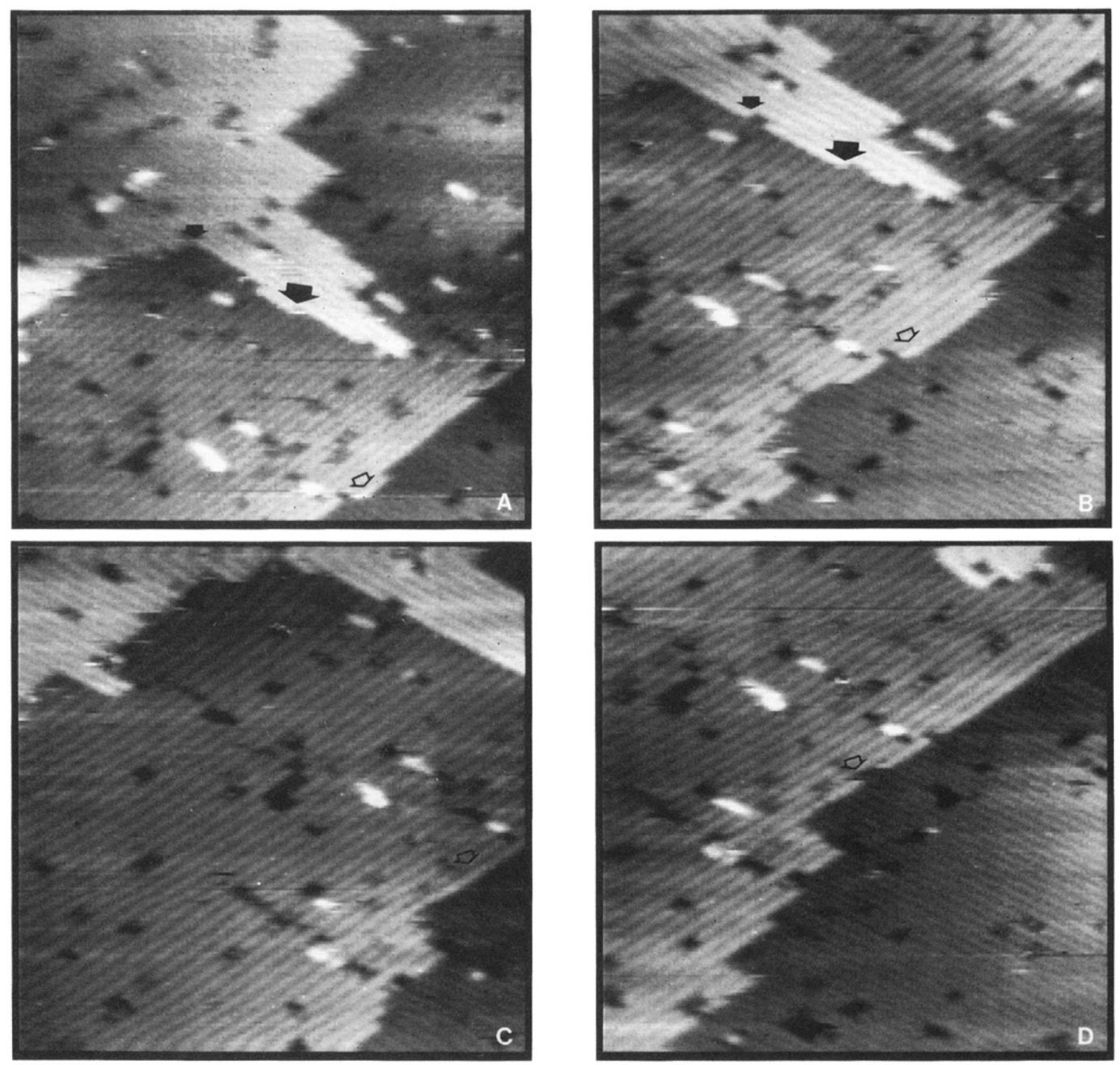

FIG. 2. (a)-(d) Several subsequent STM images of a vicinal $\mathrm{Si}(001)$ surface, at a temperature of about $725 \mathrm{~K}$. The sample bias was $-1.5 \mathrm{~V}$ and the tunneling current was $0.5 \mathrm{nA}$. The typical time lapse between successive images is about $1 \mathrm{~min}$. Scan size $30 \times 30$ $\mathrm{nm}^{2}$ 